\title{
Is Collagen an Independent Risk Factor for Breast Cancer?
}

\author{
Paola Taroni ${ }^{1,2}$, Anna Maria Paganoni ${ }^{3}$, Francesca Ieva ${ }^{4}$, Francesca Abbate ${ }^{5}$, Enrico Cassano ${ }^{5}$, Rinaldo \\ Cubeddu ${ }^{1,2}$ Antonio Pifferi ${ }^{1,2}$ \\ ${ }^{1}$ Dipartimento di Fisica, Politecnico di Milano, Piazza Leonardo da Vinci 32, 20133 Milano, Italy; ${ }^{2}$ Istituto di Fotonica e Nanotecnologie, \\ Consiglio Nazionale delle Ricerche, Piazza Leonardo da Vinci 32, 20133 Milano, Italy; ${ }^{3}$ Dipartimento di Matematica, Politecnico di Milano, \\ Piazza Leonardo da Vinci 32, 20133 Milano, Italy; ${ }^{4}$ Dipartimento di Matematica, Università degli Studi, Via Saldini 50, 20133 Milano, Italy; \\ ${ }^{5}$ European Institute of Oncology, Breast Imaging Unit, Via G. Ripamonti, 435, 20141 Milano, Italy; \\ paola.taroni@polimi.it
}

\begin{abstract}
Collagen content was estimated on 109 subjects (53 healthy and 56 with malignant breast lesions) performing time domain 7-wavelength (635-1060 nm) optical mammography. Subjects with higher age-matched collagen content (top 15\%) show higher cancer occurrence. Collagen correlates with mammographic density (recognized risk factor for breast cancer). However, top 15\% clustering based on percentage mammographic density identifies a different set of patients, potentially indicating collagen as an independent breast cancer risk factor. If confirmed on a wider population, these initial results could have huge impact on personalized diagnostics and health care systems.

OCIS codes: (170.6510) Spectroscopy, tissue diagnostics; (170.3830) Mammography; (170.6920) Time-resolved imaging; (170.5280) Photon migration.
\end{abstract}

\section{Introduction}

In developed countries, one in 8 to 10 women will suffer from breast cancer in their lifetime. Early diagnosis (size $<$ $1 \mathrm{~cm}$, no lymph node involvement) is critical to achieve a high complete cure rate. Identifying subjects at high risk is thus a key goal, as it would allow designing personalized screening/diagnostics paths on small groups, which would not be sustainable in population-wide programs. Mammographic breast density is recognized as an important risk factor for breast cancer [1] Recently, optical techniques have shown to offer a real non-invasive and potentially costeffective alternative for the assessment of breast density even at young age [2,3]. However, the origin of breast cancer is not fully understood yet, and other important risk factors may still be unidentified.

In this work, we start pursuing the ambitious goal of determining whether optical techniques can be applied to assess breast cancer risk factors directly, and not just indirectly by correlation with mammographic density. More specifically, we focus on collagen, quantified by time-resolved diffuse optical spectroscopy, as a potential independent risk factor for breast cancer [4,5].

\section{Experimental set-up and clinical study}

Clinical study

218 subjects enrolled in a study on optical mammography. For the purpose of the present investigation, the following criteria were considered i) exclusion of patients with benign lesions or not clearly classified malignant lesions (e.g. DCIS), ii) availability of recent digital mammograms for percentage breast density assessment; iii) absence of technical failures or limitations (e.g. poor signal). This identified a subset of 109 subjects: 53 healthy subjects (normal) and 56 patients bearing a malignant breast lesion (cancer).

Instrument set-up [6]

Time-resolved transmittance images were acquired at 7 wavelengths $(635,685,785,905,930,975,1060 \mathrm{~nm}) \mathrm{using}$ pulsed diode lasers and time-correlated single photon counting. The compressed breast was scanned continuously recording data every $\mathrm{mm}$. The central region of the breast, far from boundaries, was used for the assessment of the average optical properties of breast tissue.

Data analysis

Tissue composition and structure were estimated directly from time-resolved transmittance curves using a spectrally constrained global fitting procedure on the key absorber concentrations (oxy- and deoxy-hemoglobin, water, lipids, and collagen) together with the scattering amplitude $a$ and power $b$.

Two experienced radiologists assessed the percentage mammographic density using an automated procedure [7].

\section{Results and discussion}

To investigate a potential risk factor for breast cancer, in principle a long longitudinal study would be required, involving a high number of subjects to understand whether the factor, observed at a certain time, is correlated with 
the later occurrence of cancer. Such a demanding study can reasonably be designed and undertaken only once the initial hypothesis has been successfully explored on a much smaller scale. In line with the need of this preliminary step, here we try to get a first insight addressing retrospectively the question of whether or not collagen content in breast tissue is an independent risk factor. To this purpose, we make use of data collected from 218 subjects in a clinical study on the optical characterization of breast lesions and on the optical assessment of breast density. As mentioned, only a limited number of subjects (109) met the prerequisites for the present study, so, here we classify them in just two groups: normal (healthy subjects) and cancer (subjects bearing a malignant breast lesion).

Mammographic breast density is widely recognized as a strong and independent risk factor for breast cancer and we have previously shown that a non-invasive estimate of breast density can effectively be obtained with an optical index based on collagen content and on its microscopic structure (through the dependence on the slope of the scattering spectrum) [2]. Thus, collagen content can certainly provide information on risk: the question here is whether this information comes only from the correlation between collagen and mammographic density or whether collagen can provide further (independent) information on risk, being itself an independent risk factor.

The average collagen content in cancer subjects is just slightly higher than in normal subjects, but the difference is not significant based on the Mann-Whitney test. On the other hand, notwithstanding the high data dispersion, percentage density is significantly higher in cancer subjects than in normal ones $(p=0.0101)$.
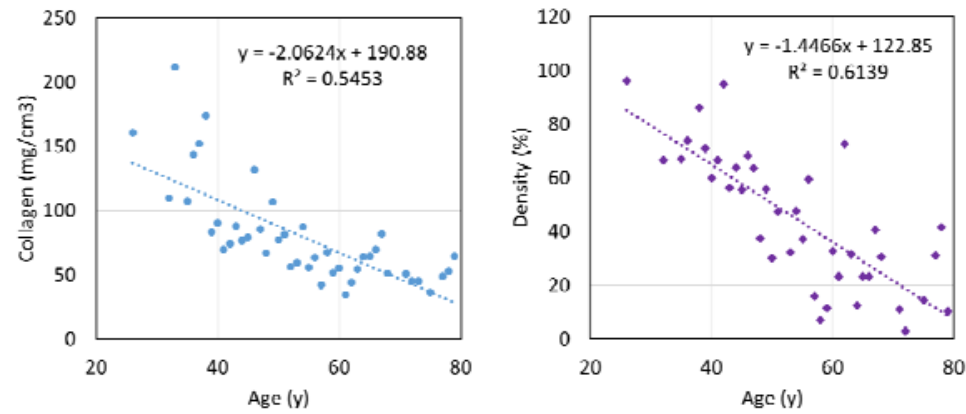

Fig. 1. Dependence of collagen content (left) and mammographic density (right) on age.

As shown in Fig. 1, on average both mammographic density and collagen content decrease with age, which is known as the strongest risk factor for breast cancer. Thus, to uncouple the effects of age, both collagen content and percentage density were divided by the corresponding theoretical age-matched value obtained by a linear interpolation on the distribution for normal subjects as a function of age. A quadratic regression, which better fits the age dependence of collagen, was also tested. Yet, the results discussed in the following did not improve significantly.
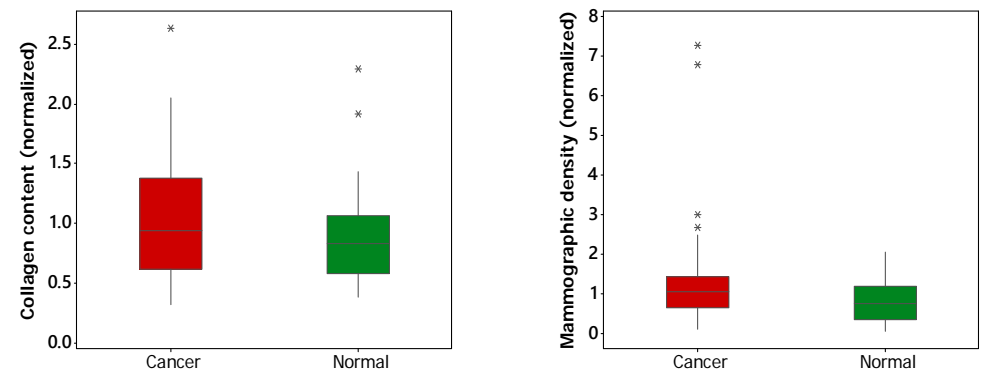

Fig. 2. Boxplot of age-normalized collagen content (left) and mammographic density (right) in cancer and normal groups.

Fig. 2 displays the boxplots of age-normalized collagen content and mammographic density in cancer and normal groups. Both collagen $(p=0.0366)$ and mammographic density $(p=0.0066)$ are significantly higher in cancer than in normal subjects. Still, collagen seems to perform worse than mammographic density, and, apparently, it might not be able to provide independent information on risk. However, it should be taken into account that, even in case our hypothesis is confirmed, only a fraction of the total population will develop cancer because of their higher collagen content. Thus, the effect could hardly be observed on average values.

Based on mammographic density, high risk subjects can be identified with BI-RADS category 4, which includes approximately $10 \%$ (or a slightly higher percentage) of the overall population [8]. Similarly, to try to identify a 
restricted subgroup at high risk for their high collagen content, we sorted subjects based on their collagen content and classified the top $15 \%$ of the ranking as "high-risk".

Table 1. Classification based on top $15 \%$ of either high collagen or high density as a marker of high risk

\begin{tabular}{c|c|c|}
\multicolumn{1}{c}{} & \multicolumn{1}{c}{ Cancer } & \multicolumn{1}{c}{ Normal } \\
\cline { 2 - 3 } High-risk & 13 & 3 \\
\cline { 2 - 3 } Low-risk & 43 & 50 \\
\cline { 2 - 3 } & &
\end{tabular}

The results (Table 1) are strictly the same for high collagen or high density: cancer patients represent $51 \%$ of the overall study population, while they are up to $81 \%$ in the high-risk category. This suggests that optical measurements are as effective as mammographic density in identifying high-risk subjects (with the advantage of absolute non-invasiveness). It is interesting to observe that the groups of high-risk subjects identified by the two techniques do not fully overlap. Actually, the 3 normal subjects that are included in the high risk group are different when collagen is considered as a risk marker instead of mammographic density.

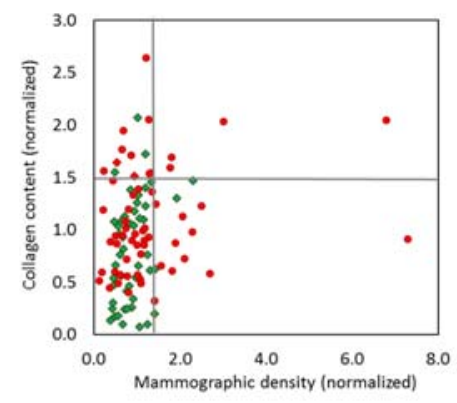

Fig. 3. Age-normalized collagen content vs mammographic density in cancer (red circles) and normal (green diamonds) groups.

Fig. 3 shows the age-normalized collagen $v s$ breast density for cancer and normal subjects. The different regions correspond to the high/low risk discrimination discussed above. The two classifications are clearly different. The number of subjects considered here is very small, but still this seems to suggest that collagen content may contain some information on cancer risk that is independent of what obtained with x-ray mammography.

\section{Conclusion}

In summary, in a small preliminary retrospective study involving 109 patients we observed that age-matched collagen content allows the identification of a group with higher cancer occurrence than in the whole study population. Further, the group of high-risk subjects identified based on collagen content does not fully overlap the one derived based on mammographic density. This implies that collagen could address a somewhat different or complementary risk factor as compared to x-rays. If confirmed statistically on larger populations, these results might have huge impact. Optical techniques could be used as an independent non-invasive mean in screening programs aiming at identifying high-risk subgroups that deserve personalized diagnostic paths. Moreover, collagen content could be exploited to develop combined risk prediction models and refine subject clustering. Finally, the noninvasive assessment of collagen can be of great interest in basic research to investigate in vivo the link between collagen and cancer onset, and the dependence of treatment response on breast composition and structure.

\section{References}

[1] V. A. Mccormack and S. Silva, "Breast Density and Parenchymal Patterns as Markers of Breast Cancer Risk : A Meta-analysis," Cancer Epidemiol. Biomarkers Prev. 15, 1159-1169 (2006).

[2] P. Taroni, A. Pifferi, G. Quarto, L. Spinelli, A. Torricelli, F. Abbate, A. Villa, N. Balestreri, S. Menna, E. Cassano, and R. Cubeddu, "Noninvasive assessment of breast cancer risk using time-resolved diffuse optical spectroscopy.," J. Biomed. Opt. 15, 060501 (2010)

[3] K. M. Blackmore, J. A. Knight, and L. Lilge, "Association between transillumination breast spectroscopy and quantitative mammographic features of the breast.," Cancer Epidemiol. Biomarkers Prev. 17, 1043-50 (2008).

[4] J. Couzin, "Breast cancer. Dissecting a hidden breast cancer risk.," Science 309, 1664-6 (2005).

[5] C. Luparello, "Aspects of Collagen Changes in Breast Cancer," J. Carcinog. Mutagen. S13, (2013).

[6] P. Taroni, A. Pifferi, E. Salvagnini, L. Spinelli, A. Torricelli, and R. Cubeddu, "Seven-wavelength time-resolved optical mammography extending beyond $1000 \mathrm{~nm}$ for breast collagen quantification.," Opt. Express 17, 15932-46 (2009).

[7] Tagliafico, G. Tagliafico, S. Tosto, F. Chiesa, C. Martinoli, L. E. Derchi, and M. Calabrese, "Mammographic density estimation: comparison among BI-RADS categories, a semi-automated software and a fully automated one.," Breast 18, 35-40 (2009).

[8] "BI-RADS® - Mammography 2013 - American College of Radiology," http://www.acr.org/quality-safety/resources/birads/mammography. 